\title{
Mobility law of dislocations with several character angles and temperatures in FCC Aluminum
}

\author{
Jaehyun Cho, Jean-François Molinari, Guillaume Anciaux* \\ Civil Engineering Institute, Materials Science and Engineering Institute, \\ École Polytechnique Fédérale de Lausanne (EPFL), Station 18, CH-1015 Lausanne, Switzerland
}

\begin{abstract}
We study the mobility law of dislocations in aluminum as an important building block for the development of a multiscale method that couples an atomistic model with discrete dislocation dynamics in 3d (e.g., CADD3d). Straight dislocations of arbitrary character angles are modeled with classical molecular dynamics at several temperatures. The obtained mobility results are analyzed and validated by comparisons to theoretical models. A critical velocity parameter identified by the analytic models is correlated to the material dispersive nature. We revisit the interpretation of this constant by considering character angles that were not studied previously. Finally, the obtained mobility law is implemented and employed in the discrete dislocation dynamics simulation of a dislocation loop. Our results highlight the importance of including several angles when constructing the mobility law to produce consistent results.
\end{abstract}

Keywords: Mobility law; Dislocation; CADD; Multiscale Modeling

\section{Introduction}

Dislocations are line defects composed of mis-connected atomic arrangements. These dislocations move in several ways including gliding, climbing and grouping as forests $[1$, 2], and consequently impact material properties during plastic deformations. During the last decades, many experiments and computer simulations have been conducted to study dislocation motions, revealing several interesting mechanisms as consequence of dislocation dynamics [3-5] (e.g., size effect of material strength).

One of the computational methods is classical molecular dynamics (MD). By displacing atoms, dislocations can be nucleated, and their dynamics follow force fields and material potentials. Unfortunately, state-of-the-art MD simulations can only track dislocations within systems of small length scales. Therefore, when employing MD, the simulations of long-range interactions between dislocations at scales above micrometers are out of reach [4]. Another computational approach, discrete dislocation dynamics

\footnotetext{
*Corresponding author: guillaume.anciaux@epfl.ch

Email addresses: jaehyun.cho@epfl.ch (Jaehyun Cho), jean-francois.molinari@epfl.ch (Jean-François Molinari), guillaume.anciaux@epfl.ch (Guillaume Anciaux)

Preprint submitted to Elsevier

October 24, 2016
} 
(DD), represents coarse-grained dislocation structures [6], and can consequently simulate dislocation dynamics at larger length and time scales than MD. This feature enables DD simulations to provide results, which are comparable to experiments above micron scales [7]. The successful implementation of DD needs two ingredients. First, some appropriate material parameters should be employed, e.g., mobility law $[8,9]$ and core energy $[10,11]$. These can be collected from theories, experiments or MD simulations. Second, initial conditions are required with regards to dislocation nucleations $[12,13]$. These conditions are often adhoc [14]. Again, it must be noted that MD implicitly considers the general and complex interactions between dislocations including their nucleations as opposed to DD.

Consequently, a multiscale simulation coupling MD, where dislocation nucleations are naturally accounted for, with DD to enlarge simulation box length scales, offers new possibilities for modeling plasticity across length scales. This multiscale approach has been successfully implemented in a two-dimensional system (CADD2d) $[15,16]$. The authors are developing a $3 \mathrm{~d}$ extension named CADD $3 \mathrm{~d}^{1}$ of the pre-cited coupling method. Unlike CADD2d, 3d dislocations with arbitrary character angles can simultaneously exist in $\mathrm{MD}$ and $\mathrm{DD}$ regions. In this situation, two different representations are necessary for any single dislocation: an atomic structure and a set of nodes connected by straight lines in MD and DD, respectively. In order to move these two representations as a single physical dislocation, a proper communication must be enforced at the interface [17]. This communication can be achieved through respective boundary conditions, e.g., core templates [18] and a dislocation detection algorithm [19]. We emphasize that the correct communication during dislocation dynamics can only be achieved when the two dislocation representations move at the same pace. This can be feasible by employing an accurate-mobility law in DD.

In a pioneering work, Nabarro [20] studied dislocation mobility with a continuum approach and showed that dislocations should not propagate at a velocity larger than the shear wave speed. Much below this forbidden velocity, dislocation motions are dragged by a phonon viscous force in a crystal lattice. This drag force is proportional to temperature [21-23]. Eshelby [24] found a distinct supersonic solution to the equation of a dislocation motion and introduced the idea that during motion of a dislocation, both sub-sonic and super-sonic waves can be emitted depending on the dislocation velocity. This mixed-wave dispersion brings another dislocation drag mechanism, which explains how dislocations can loose energy by radiations, besides the phonon viscous force. The transition from phonon-viscous dragging to damping due to dispersive nature happens when the dislocation velocity becomes larger than a critical velocity $v_{0}$. Later, this critical transition-velocity has also been analyzed thanks to lattice dynamics [25-28] based on a Frenkel-Kontorowa model [29].

Dislocation mobilities can also be investigated using MD simulations [30-33]. For example, Kang et al. [30] recently conducted computations of the BCC Peierls limits for various angles to derive a kink-driven mobility law. To the best of our knowledge, direct measurements of mobilities with MD remain limited to very few orientations (edge/screw for FCC [31-33] and screw/M111 for BCC [30]). Indeed, for FCC materials the mobility law employed in DD models is assumed to be a monotonically varying function between

\footnotetext{
${ }^{1}$ Swiss national science foundation project conducted by three principal investigators, W.A. Curtin, J.F. Molinari and G. Anciaux.
} 
the edge and screw dislocation mobilities [8, 34, 35]. However, we will show that it is not the case. This is an important contribution of the present paper, as it is known that the character angle impacts macroscopic plasticity in FCC crystals, for instance through cross-slips $[1,31,36]$. Consequently, having the correct mobilities for multiple orientations is particularly crucial.

This article is organized as follows: in Section 2, the CADD3d coupling framework is briefly recalled to further motivate the study of mobility law. Then, dislocation mobilities for various mixed angles and temperatures are extracted from atomistic simulations in Section 3. In Section 4, the obtained mobility results are fitted to an Eshelby-Olmsted [24, 32 ] equation, and the outcome is discussed. In particular, we comment on the origin of one important fitting parameter, called the critical velocity, which identifies a change in the dislocation damping mechanisms. In Section 6 , we finally construct a mobility law issued from these findings and use it in DD simulations.

\section{Background: Mobility law in CADD3d}

In two-dimensional CADD $[15,16]$, only infinitely straight dislocations can be modeled because of the plane-strain restrictions. Thus, the dislocations, here defined pointlike, can only exist in the MD or in the DD domains but never in both at a time. Consequently, the process of dislocation passing from MD to DD regions is simplified. When a MD dislocation is detected near the MD boundary, a corresponding DD dislocation is inserted in the DD region. However, this procedure cannot be applied when a three-dimensional system is considered. This issue is well illustrated by a dislocation loop which would be partly in the MD domain and partially in the DD domain, thus having two different representations. Such a hybrid dislocation, with a coupling that crosses the interface between MD and DD domains, needs to glide smoothly without artifacts.

Figure 1 illustrates the dynamical motion of the hybrid dislocation. Time is explicitly integrated by Verlet integration and Euler-forward schemes in MD and DD domains, respectively, and the $\mathrm{MD}$ and $\mathrm{DD}$ dislocations move ignoring the coupling constraints between two coupling steps $\left(t_{n-1}\right.$ and $\left.t_{n}\right)$. Then, the atoms in a pad region ${ }^{2}$ are positioned in agreement with the dislocation position provided by DD nodes. This operation uses specific core structures that were pre-computed in offline calculations [18]. Reciprocally, the dislocation core is detected [19] in the MD region and allows to update the DD boundary condition [17].

If the coupled models disagree on the evolution of its dislocations, then a bi-material interface is effectively simulated, with an artificial pinning of the hybrid dislocation to be expected. In DD dynamics, dislocation nodes react to resolved shear stresses through the so-called mobility law $M$, which is defined uniquely for each material type [8]. In the case of FCC crystalline materials, dislocations dissociate into two partial dislocations [1], which enforces planar core structures and prevents climbing mechanisms [37]. Consequently, the only variables for the mobility law of FCC materials are the character angle $\theta$ and the temperature $\mathrm{T}[8]$. In the following, we therefore compute and analyze the influence of these parameters thanks to atomistic simulations of straight dislocations with various character angles and temperatures.

\footnotetext{
${ }^{2}$ Interface region with a finite thickness between MD and DD domains. A more detailed description can be found in $[17,18]$.
} 


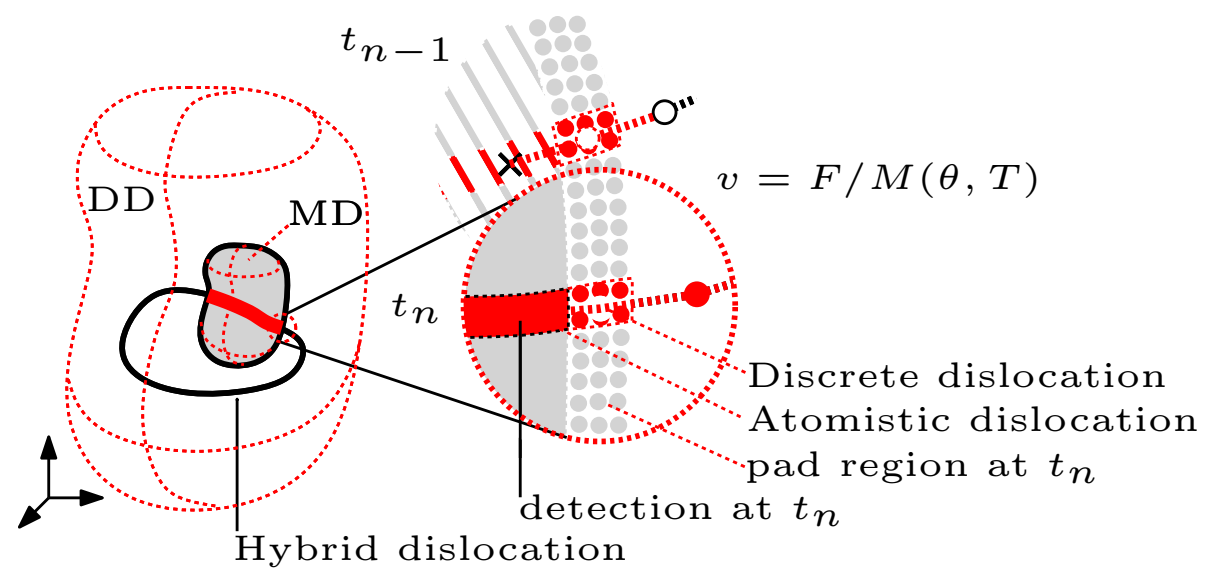

Figure 1: Schematic of a hybrid dislocation dynamics in CADD3d. Interface between MD and DD domains is zoomed, and shown in a (2D) picture at each coupling step $\left(t=t_{n-1}\right.$ or $\left.t=t_{n}\right)$. The MD and DD dislocations are coupled and communicate with each other by reciprocal boundary conditions through the atoms in pad region [18] and the dislocation detection [17, 19]. Between the two coupling steps, the MD dislocation glides following atomistic force fields derived from material potentials, and the DD dislocation travels following the mobility law $M(\theta, T)$.

\section{Atomic dislocation modeling}

The schematic modeling of a single dislocation is shown in Figure 2. At the center of the simulation box, a dislocation with an arbitrary character angle $\theta$ is inserted. We choose in total 8 -character angles from screw $\left(0^{\circ}\right)$ to edge $\left(90^{\circ}\right)$ dislocations. Periodic boundary conditions (PBC) are imposed in the dislocation line direction $(x)$ and in the gliding direction $(z)$. We choose the Mendelev et. al. aluminum EAM/FS potential [38], which describes well the stacking fault energies obtained from DFT calculations [18]. The details of the modeling procedures, the character angle selections, the dislocation core structures (at $0 K$ ) and the stacking fault energies for the selected potential have been extensively discussed by Cho et. al. [18]. We select three different temperatures $\mathrm{T}=100 \mathrm{~K}$, $200 K$ and $300 K$ by relaxing the atoms using a Langevin thermostat [39]. Furthermore, we also expand the simulation box by $0.26 \%, 0.48 \%$ and $0.7 \%$ for the $100 K, 200 K$ and $300 K$ cases, respectively, during the relaxations in order to correct for pressures due to thermal expansions.

In order to initiate the dislocation motion after the relaxation, a shear loading is applied on the top and bottom free surfaces in the direction of the Burgers vector $\vec{b}=$ $\frac{1}{2}[1 \overline{1} 0]$. The shear loads have been chosen between $50 \mathrm{MPa}$ to $1000 \mathrm{MPa}$. As the shearing occurs, the dislocation glides smoothly on the slip plane and creates heat. In order to keep the system at a constant temperature, Langevin thermostats with a damping constant $\tau=10000 \Delta t(10 \mathrm{ps})$ are used close to the top and bottom surfaces. A large distance $(60 \AA)$ separates the thermostat regions from the dislocation core to limit the artificial frictional forces acting on the core region. Consequently, the dislocation can glide in the direction $z$ infinitely (thanks to PBC conditions), while keeping globally constant the targeted temperature. 


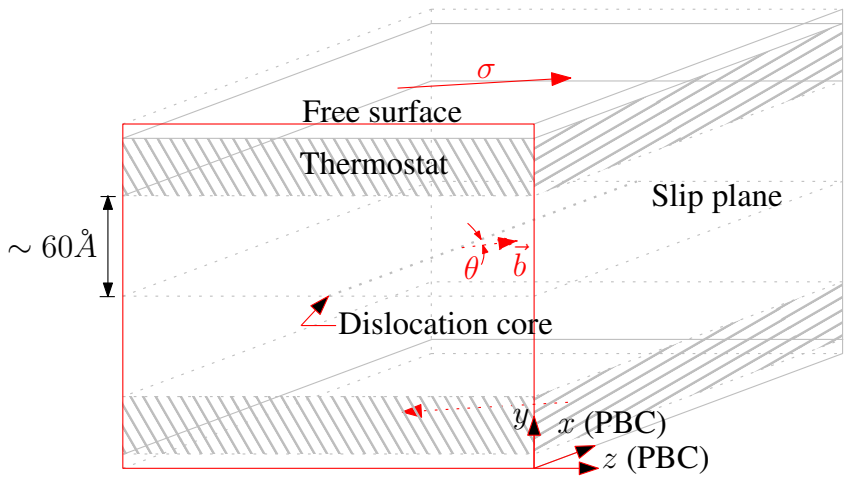

Figure 2: Schematic modeling of a straight dislocation with an arbitrary character angle $\theta$. The length of simulation box in $y$ axis is $\sim 200 \AA$, while the dimensions in $x$ and $z$ axes vary around $70 \AA$ and $350 \AA$, respectively, with the lattice orientations determined by the character angle $\theta$ [18]. A finite temperature is chosen by Langevin thermostats and a shear stress is applied on the free surfaces in the direction of the Burgers vector $\vec{b}=\frac{1}{2}[1 \overline{1} 0]$.

Figure 3 (a) shows the temperature evolution around the slip plane for the $90^{\circ}$ dislocation and for several shear stresses at $100 \mathrm{~K}$. There are fluctuations during dislocation dynamics which decay quickly. When the dislocation stabilizes, the absolute magnitudes of the fluctuations are within $0.1 \%$ of the target temperature $(100 \mathrm{~K})$. The temperature imprecision remains within $10 \%$ (the maximum is $106 \mathrm{~K}$ for the largest applied stress considered), which is acceptable. Figure 3 (b) shows the velocity variations of $49.107^{\circ}$ and $90^{\circ}$ dislocations for an applied shear stress $\sigma=300 \mathrm{MPa}$ at two different temperatures $\mathrm{T}=100 \mathrm{~K}$ and $300 \mathrm{~K}$. At time $t=0 \mathrm{ps}$, the dislocation escapes the stationary state, and starts to accelerate. At the beginning of gliding ( $5 \mathrm{ps}<t<30 \mathrm{ps})$, the dislocation dynamics are influenced by waves due to the sudden shear loading applied on the top and bottom surfaces. After 30ps, the dislocation glides at a globally constant velocity. By taking the average values at the steady-state dislocation velocity regimes, the variation of dislocation velocities can be obtained. Moreover, the stress field around the dislocation core (effective stresses $\sigma_{e f f}$ ) is measured. The evolution of these effective stresses is shown in Figure 3 (c) for several applied shear stresses. We see that the applied stress $\sigma_{a p p}$ is not coinciding with the effective stress $\sigma_{e f f}$ acting on the dislocation. The difference is due to the thermostat's friction force. More precisely, the dislocation motion generates heat proportionally to the dislocation velocity. Accordingly, the viscous forces sustaining the targeted temperature are proportional to that velocity. This is confirmed with Figure 3 (d) which shows the relationship between the magnitudes of the stress differences as function of the dislocation velocity: a linear correlation clearly applies. Finally, we can construct the relationship between dislocation velocities and the effective stresses. Figure 3 (e) shows this relationship for the $49.107^{\circ}$ and $90^{\circ}$ dislocations at $100 \mathrm{~K}$ and 300K. The simulations reveal that:

1. The dislocation velocities first increase linearly with the effective stress, and progressively saturate due to an increase of the drag force.

2. The dislocations in the hot systems $(300 \mathrm{~K})$ glide slower than the dislocations in the cold systems $(100 \mathrm{~K})$. 

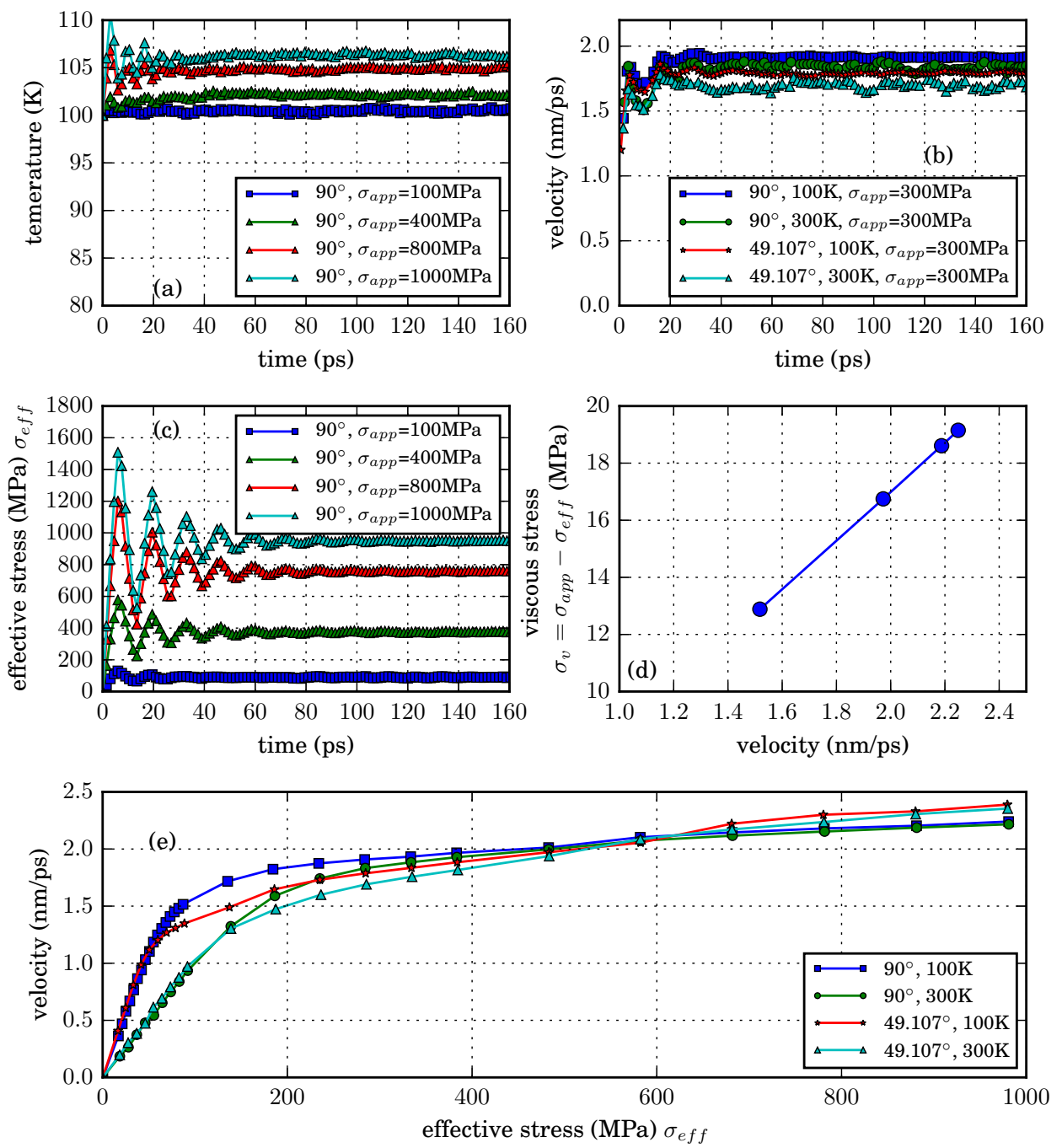

Figure 3: (a) Evolution of temperatures at the vicinity of the slip plane of the edge dislocation with various shear stresses. The target temperature is $100 \mathrm{~K}$. (b) Velocity of dislocations $\left(49.107^{\circ}\right.$ and $\left.90^{\circ}\right)$ at $100 \mathrm{~K}$ and $300 \mathrm{~K}$ under $300 \mathrm{MPa}$. (c) Variations of effective stresses around the slip plane during edge dislocation motion. (d) Differences between the applied and effective stresses as function of the dislocation velocity. (e) Variations of steady-state velocities for $49.107^{\circ}$ and $90^{\circ}$ dislocations as a function of shear force at $100 \mathrm{~K}$ and $300 \mathrm{~K}$. 
3. This temperature effect is more important in the linear regime (for small applied shear) than in the saturation regime.

4. The strength of this temperature effect depends on the character angle.

These observations are consistent with MD simulations in several publications [3133] for screw $\left(0^{\circ}\right)$ and edge $\left(90^{\circ}\right)$ dislocations, and can be rationalized by theoretical models [20-24]. In the following section, we will investigate the variation of the mobility law with respect to the character angle and compare it with the aforementioned models.

\section{Eshelby-Olmsted approach}

Olmsted et. al. [32] recently studied dislocation mobility laws using atomistic simulations, and showed that their results were confirming previous theoretical observations [2024]. More precisely, they fitted their numerical findings to the following phenomenological equation [32]:

$$
\sigma_{e f f}= \begin{cases}A \mathrm{~T} v_{d} & \text { if } v_{d} \leq v_{0} \\ A \mathrm{~T} v_{d}+D\left(v_{d}-v_{0}\right)^{3 / 2} & \text { if } v_{d}>v_{0}\end{cases}
$$

with $v_{d}$ the dislocation velocity, $\sigma_{\text {eff }}$ the effective shear stress, $\mathrm{T}$ the temperature, and $A$, $D$ two fitting constants. This equation accounts for the transition of dislocation damping mechanisms as a dependence on the dislocation velocity $v_{d}$. When the dislocation velocity $v_{d}$ is smaller than the critical velocity $v_{0}$, only the phonon viscous term $A \mathrm{~T} v_{d}$ is considered. The dislocation mobility in this linear regime $\left(v_{d} \leq v_{0}\right)$ can be described by the damping constant $A$ times the corresponding temperature T. For $v_{d}>v_{0}$, an additional damping effect occurs due to supersonic wave emissions. Eshelby [24] showed that this new contribution should increase non-linearly, taking the form $\left(v_{d}-v_{0}\right)^{3 / 2}$, and thus leads to the asymptotic dislocation velocities that we also observed. Following his argument, the constant $D$ should not depend strongly on the temperature T.

Figure 4 shows all the relations between dislocation velocity and the effective stress that we have simulated. The data computed from MD simulations are denoted by dots, while the solid curves are fits using Equation 1. For each character angle, a single parameter of $\mathrm{A}$ and $v_{0}$ is fitted, and three different constants $\mathrm{D}$ are used to fit the results of three temperature cases. The results show a generally good agreement with the simulated data: we have a match within a $\sim 7 \%$ error for all the character angles and temperatures investigated. However, Equation 1 yields a strong transition between the two different velocity regimes (damping mechanisms), and does not capture well the smooth transitions as observed in the MD results.

Figure 5 (a) shows the fitting parameter $A|\vec{b}|$, where $|\vec{b}|$ is the magnitude of Burgers vector, as function of the character angle $\theta$. Screw-like dislocations $\left(0^{\circ} \text { and } 60^{\circ}\right)^{3}$ show the highest damping values $A|\vec{b}|$ implying large phonon viscous forces. According to experiments $[22,23,40]$, the range of damping constant $A|\vec{b}|$ is between 1.8 and $7.0 \times 10^{-8}$ $\left[\mathrm{Pa} \cdot \mathrm{s} \cdot \mathrm{K}^{-1}\right]$. Other atomistic simulations $[32,33]$ provided damping parameters between 3.7 and $4.5 \times 10^{-8}\left[\mathrm{~Pa} \cdot \mathrm{s} \cdot \mathrm{K}^{-1}\right]$, and 6.0 and $7.5 \times 10^{-8}\left[\mathrm{~Pa} \cdot \mathrm{s} \cdot \mathrm{K}^{-1}\right]$ for edge and screw dislocations, respectively. This reveals that the damping parameters obtained from our

\footnotetext{
${ }^{3}$ The two dislocations are constructed based on the same family of lattice orientation associated with screw dislocations, $\mathrm{x}:\{110\} \mathrm{y}:\{111\}$ and $\mathrm{z}:\{112\}$.
} 

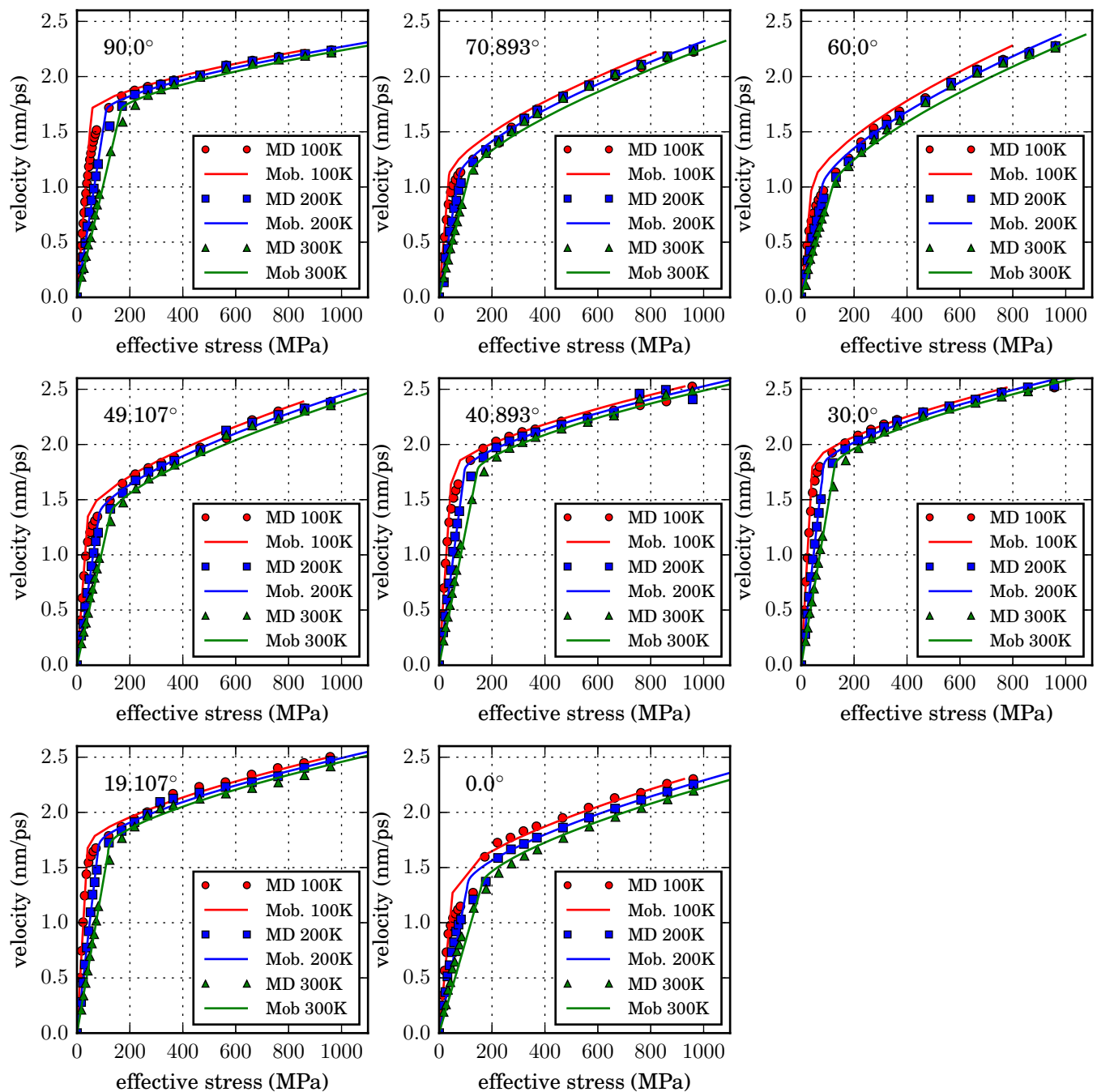

Figure 4: Dislocation mobility for various character angles and temperatures. The MD results are described by the markers and the fits using Equation 1 are depicted by the solid lines. For the $100 \mathrm{~K}$, $200 \mathrm{~K}$ and $300 \mathrm{~K}$ cases, the simulations and fits are denoted with the red, blue and green colors. The critical velocities $v_{0}$ are notified by the black-dashed lines. 

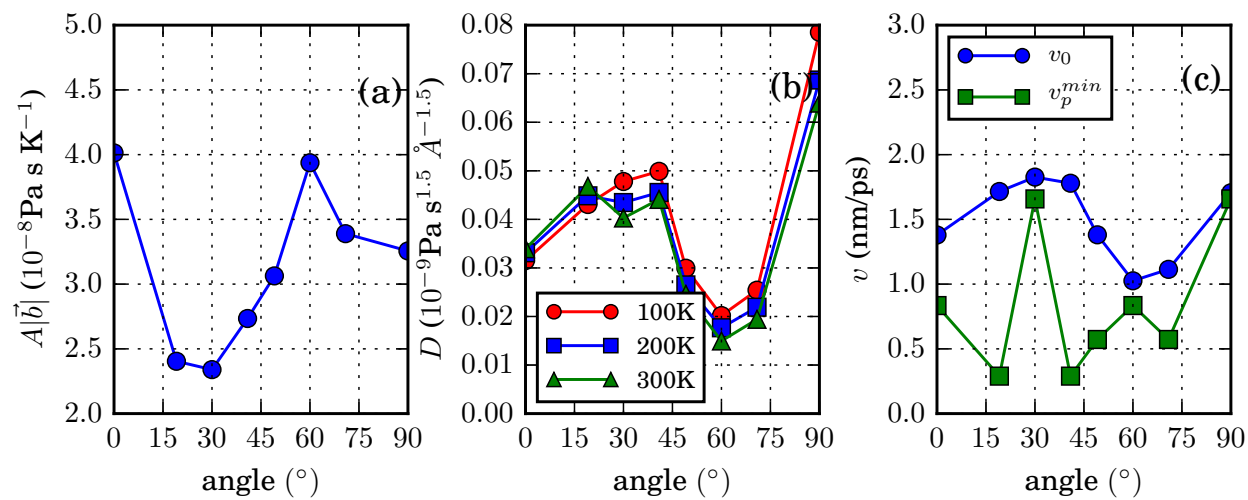

Figure 5: (a) Variation of the damping parameter $A$ multiplied by the magnitude of Burgers vector $\mid \vec{b}$ for various character angles. (b) Variations of damping constant $D$ with respect to character angle. The constants for $100 \mathrm{~K}, 200 \mathrm{~K}$ and $300 \mathrm{~K}$ cases are represented by red circle, blue square and green triangle markers, respectively. (c) The fitted critical velocity $v_{0}$ and the minimum phase velocity $v_{p}^{\text {min }}$ in the dislocation propagation direction of various character angles.

atomistic simulations are within the range of reported values. Figure 5 (b) shows the fitting parameters $D|\vec{b}|$ and $v_{0}$ for the studied character angles $\theta$ and temperatures T. As could be granted to the Eshelby analysis, the fitted parameter $D$ depends weakly on temperature. Concerning the critical velocity $v_{0}$, Marian and Caro [41] recently argued that it can be defined from the reciprocal space structure, and more precisely from the crystalline dispersion curves. This transition is thought to be strongly correlated to the minimum phase velocity $v_{p}^{\text {min }}$ in the dislocation propagation direction [41]. This last argument seems not general enough to explain the value of $v_{0}$ for every mixed angle, as can be observed from Figure 5 (c). In the following section, we discuss the link between the critical velocity $v_{0}$ and the dispersion relation.

\section{Discussion: critical velocity}

Many authors $[24-26,33,41]$ have discussed the existence of the critical velocity $v_{0}$. A lot of efforts aimed at understanding its origin by using lattice dynamics [25-28] based on a Frenkel-Kontorowa model [29]. All of these previous studies identified critical regions in the reciprocal lattice, which can be described by the equation:

$$
\omega(k)=k v_{d}
$$

with $\omega(k)$ the time frequency associated with the wave vector $k$ and $v_{d}$ the dislocation velocity. As Eshelby [24] noted, when $\frac{\omega(k)}{k}=v_{d}$ then the wave equation can flip from a rapidly decaying solution to an oscillatory one, which changes completely the damping mechanism. The simple interpretation is that when the dislocation moves faster than the phase velocity $v_{p}(k)=\frac{\omega(k)}{k}$, supersonic modes are created, and these waves radiate energy. 

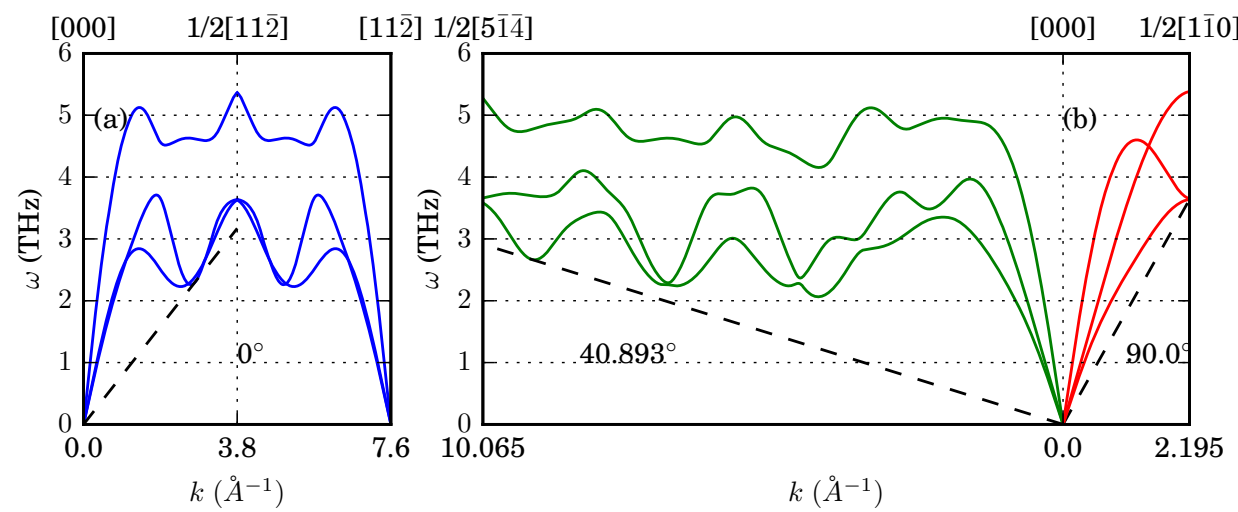

Figure 6: Dispersion curves in the propagation direction of screw $0^{\circ}$, mixed $40.893^{\circ}$ and edge $90^{\circ}$ dislocations. The three phonon modes $(\omega)$ exist for each wave number $k$. For each angle, the minimum phase velocity $v_{p}^{\text {min }}$ corresponds to the slope of the black dashed line.

To apply Eshelby's argument on our work, we need the 3 d dispersion relations $\omega(k)$ for the selected potential. These relations can be calculated by using the dynamical matrix, which is derived from the equation of motion [42]. To compute this matrix [43], a set of molecular dynamic simulations considering the fifth nearest neighbor atoms have been employed. The computed dispersion relations have been validated in specific directions by comparing to the open source OpenKIM [44]. Figure 6 shows the curves $\omega(k)$ for screw $0^{\circ}$, mixed $40.893^{\circ}$ and edge $90^{\circ}$ dislocations directions. For each of these directions, the three dispersion branches are presented.

Marian and Caro [41] studied dislocation mobilities of edge and screw dislocations, and argued that minimum phase velocities $v_{p}^{\text {min }}$ computed from these dispersion curves correspond to the critical velocities $v_{0}$ used in Equation 1. For example, a screw $0^{\circ}$ dislocation propagates in the $[11 \overline{2}]$ direction, and the three dispersion branches in that direction are shown in Figure 6 (a) by the blue curves. We see that these dispersion curves are periodic over an interval $L$ (depending on $\theta$ ), so it is natural to use $L / 2$ to compute $v_{p}^{\text {min }}$ which leads to the formula:

$$
v_{p}^{\min }=\min \left\{v_{p}(k)=\frac{\omega(k)}{k}, \quad k \in[0, L / 2]\right\}
$$

The computed $v_{p}^{\text {min }}$ for each angle corresponds to the slope of the black dashed line as shown in Figure 6 . The variations of $v_{p}^{\text {min }}$ with character angles are shown in Figure 5 (c) by the green square-dot line. When compared to $v_{p}^{\min }$ and $v_{0}$, we conclude that Marian and Caro [41] argument works well for edge-like $\left(30^{\circ}\right.$ and $\left.90^{\circ}\right)$ and for screw-like $\left(0^{\circ}\right.$ and $\left.60^{\circ}\right)$ dislocations, but it fails to predict the critical velocities $v_{0}$ for the other mixed angles.

This can be rationalized by considering crystallography as discussed in Cho et. al. [18]. The size of the minimal periodic unit cell varies with the character angle. When the axes of the FCC lattice are aligned with the $x, y, z$ directions, the minimal lattice 
employed is the FCC primitive unit cell. For completely arbitrary angles, the size of the minimal lattice changes, and there are angles (irrational) for which the periodicity length is infinity. There is a similar variation of the periodicity length in the reciprocal space, which brings the following definition problematic: the pulsation $\omega(k)$ is a necessarily bounded quantity $\left(\omega(k)<\omega^{\max }\right)$ such that:

$$
0 \leq \frac{\omega(k)}{k} \leq \frac{\omega^{\max }}{k}
$$

And consequently:

$$
0 \leq v_{p}^{\min } \leq \min \left\{\frac{\omega^{\max }}{k}, \quad k \in[0, L / 2]\right\}=\frac{\omega^{\max }}{L / 2}
$$

The immediate consequence is that for a large periodicity length $L$ of the reciprocal space, $v_{p}^{\min }$ becomes arbitrarily small. However, up to our knowledge, the transition velocity $v_{0}$ does not present such a behavior for any angle.

In reality, the assumption that excited phonons exist only in the dislocation propagation direction is incorrect. After all, the motion of atoms at the dislocation core is mainly along the Burgers vector $\vec{b}$ with a complication due to the separation into Shockley partials [1] which leads to all sorts of wave propagation directions. Consequently, the assumption of unidirectionality suggested by Marian and Caro [41] is insufficient to predict the critical velocities for general mixed dislocations in FCC crystalline materials.

\section{Implementation of mobility law}

The obtained dislocation mobility law obtained now is implemented into a discrete dislocation dynamics (DD) simulator, which is the well-known ParaDis software package $[34,45]$. In order to describe the effects of the mobility law on dislocation dynamics, we simulate dislocation loops with three different mobility modules. The first one is implemented in the present version of ParaDis. Currently, the mobility module of ParaDis uses two damping parameters $\mathrm{B}_{90}$ and $\mathrm{B}_{0}{ }^{4}$ for edge $90^{\circ}$ and screw $0^{\circ}$ character angles, respectively, and interpolates linearly between these two constants to get damping values for mixed angles [6]. With this strategy, the nodal velocity increases linearly with shear loading, and the saturation regime is not considered. The second module consists in exploiting the obtained parameters $\left(\mathrm{A}_{90}, \mathrm{D}_{90}\right.$ and $\left.\mathrm{A}_{0}, \mathrm{D}_{0}\right)$ of edge $90^{\circ}$ and screw $0^{\circ}$ dislocations. The mobility law for a mixed angle is constructed by linear interpolation between the edge and screw parameters. The last case uses the (proposed) complete mobility law, which accounts for the 8-intermediate angles considered in this work. This law allows us to refine the interpolated mobilities for the mixed angle dislocations.

Figure 7 shows the computational domain employed to model a single dislocation loop. This dislocation loop is inserted onto the centered slip plane. Anisotropic aluminum material properties justify the initial ellipsoidal shape of the dislocation loop. The size of the ellipsoid is small when compared to dislocation loops experimentally observed in aluminum. However, the simulation setting employed here aims at highlighting the

\footnotetext{
${ }^{4}$ According to our proposed mobility law, $\mathrm{B}_{\theta}$ is corresponding to $\mathrm{A}_{\theta} \mathrm{T}$.
} 


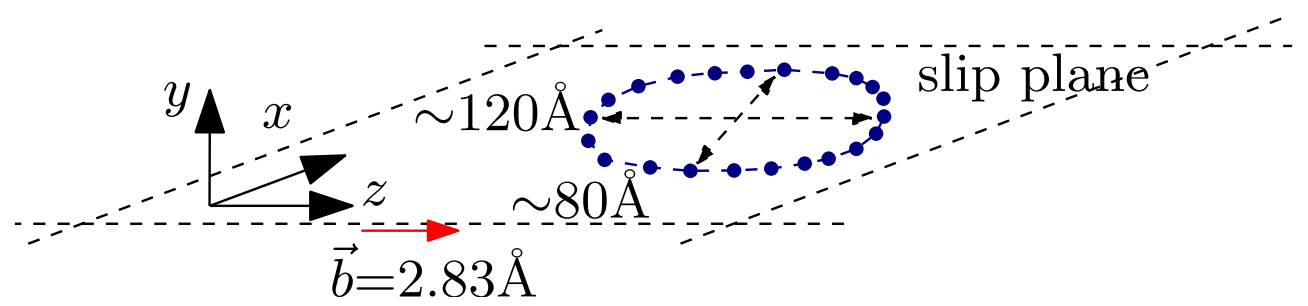

Figure 7: Schematic modeling of a dislocation loop in discrete dislocation simulation. Infinite boundary conditions are employed in all directions. A constant shear stress $\sigma_{y z}=700 \mathrm{MPa}$ activates the dislocation loop to expand.
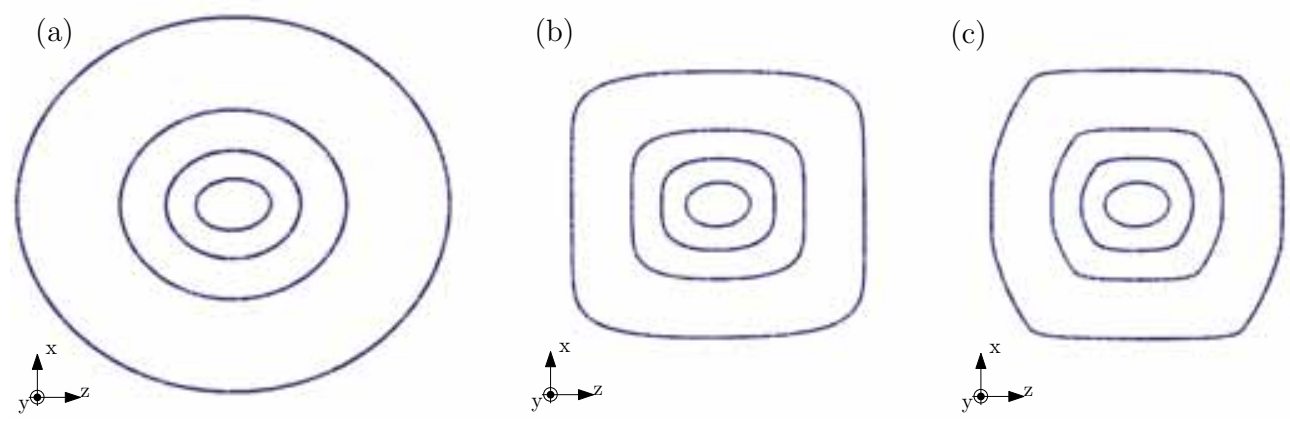

Figure 8: Snapshots of dislocation loop dynamics at 0, 2.5, 5 and 10ps with three different dislocation mobility laws: (a) A linear relation $\left(\mathrm{A}_{90}\right.$ and $\left.\mathrm{A}_{180}\right)$ between dislocation velocity and shear loading is considered and interpolated linearly from edge $\left(90^{\circ}\right)$ to screw $\left(0^{\circ}\right)$ angles. (b) The linear and supersonic asymptotic regimes are considered by considering the mobility parameters $\mathrm{A}_{90}, \mathrm{D}_{90}$ and $\mathrm{A}_{0}, \mathrm{D}_{0}$ for edge $\left(90^{\circ}\right)$ and screw $\left(0^{\circ}\right)$ dislocations. Intermediate angles are interpolated linearly from edge to screw. (c) The new mobility law proposed, which accounts for the linear and supersonic regimes and a total of 8-mixed angles.

influence of the mobility law on dislocation dynamics, which we believe applies also for larger dislocation loops. In order to expand this small loop, a shear stress $\sigma_{y z}=$ $700 \mathrm{MPa}$ is applied, which is large enough to avoid any shrinking of the dislocation loop. The trapezoidal integration scheme is applied with a timestep of 1 femto-second $\left(10^{-15}\right.$ second).

Figure 8 shows top-view snapshots of the slip plane at 0ps, 2.5ps, 5ps and 10ps with the three different dislocation mobility strategies described above. Comparing the results shown in Figures 8 (a) and (b), we can conclude that the current DD engine cannot correctly calculate the dislocation dynamics. As the loops expand, the effective stresses acting on DD segments increase due to the decay of self interactions, and describing the non-linear behavior of the mobility low becomes important. This effect is comparable with the one described by Kang et al. [30] for BCC materials.

Consequently, two different dislocation structures are finally observed at 10ps. Also, when comparing Figures 8 (b) and (c), it is clear that the mobility law accounting for intermediate angles produces large differences in the loops dynamics. In the latter case, 
the ellipsoidal loop becomes an octagon with three distinct facets in the direction of edge, screw, and mixed $60^{\circ} \sim 70^{\circ}$ dislocations. This contrasts with the rounded rectangle aligned with edge and screw dislocation directions in the former case. Arguably, the octagon shape is a more favorable dislocation loop structure since these shapes have been observed in experiments [46] dealing with FCC aluminum. These three simulation results show the necessity of defining the anisotropic mobility law not only for discrete dislocation dynamics but also for the future CADD3d development in order to minimize a source of error over the global dislocation network evolution (described in Section 2).

\section{Conclusion}

In this article, the mobilities of straight dislocations in FCC aluminum were studied using atomic simulations (MD) at several temperatures and for various character angles. The obtained velocity-effective_stress curves were successfully fitted to the EshelbyOlmsted equation. We could distinguish the two velocity regimes following the damping mechanisms due to phonon viscosity and (supersonic) wave radiations. The phonon viscosity regime is characterized by the constant $A$ that was shown to be in good agreement to other works in the literature for edge and screw dislocations. The damping constant $D$, relevant to the radiation regime, depends weakly on the temperature as was expected from Eshelby's theory. These two regimes are separated by the critical velocity $v_{0}$. We could show that $v_{0}$ cannot simply be defined as the minimum phase velocity $v_{p}^{\min }$ in the propagation direction of the dislocation. Finally, the obtained mobility law, which is valid for several character angles, has been implemented in a discrete dislocation dynamic engine. The results show the large impact of having accurate mobilities on the dislocation dynamics. This work will be employed in the future CADD3d method.

\section{Acknowledgments}

The support of the Swiss national science foundation is gratefully acknowledged (grant no. 200021-140506/1).

\section{References}

[1] JP Hirth and J Lothe. Theory of Dislocations. Krieger Publishing Company, 1992.

[2] C Kahloun, G Monnet, S Queyreau, LT Le, and P Franciosi. A comparison of collective dislocation motion from single slip quantitative topographic analysis during in-situ $\{\mathrm{AFM}\}$ room temperature tensile tests on cu and fe crystals. International Journal of Plasticity, 84:277 - 298, 2016.

[3] JR Greer, CR Weinberger, and W Cai. Comparing the strength of FCC and BCC sub-micrometer pillars: Compression experiments and dislocation dynamics simulations. Materials Science and Engineering: A, 493(12):21-25, 2008. Mechanical Behavior of Nanostructured Materials, a Symposium Held in Honor of Carl Koch at the TMS Annual Meeting 2007, Orlando, Florida.

[4] Y Gao, JR Carlos, DR Tramontina, and HM Urbassek. Comparative simulation study of the structure of the plastic zone produced by nanoindentation. Journal of the Mechanics and Physics of Solids, 75(0):58 - 75, 2015.

[5] JY Kim, D Jang, and JR Greer. Crystallographic orientation and size dependence of tensioncompression asymmetry in molybdenum nano-pillars. International Journal of Plasticity, 28(1):46 $52,2012$.

[6] VV Bulatov and W Cai. Computer Simulations of Dislocations. Osmm Series. OUP Oxford, 2006.

[7] M Huang, L Zhao, and L Tong. Discrete dislocation dynamics modelling of mechanical deformation of nickel-based single crystal superalloys. International Journal of Plasticity, 28(1):141 - $158,2012$. 
[8] W Cai and VV Bulatov. Mobility laws in dislocation dynamics simulations. Materials Science and Engineering: A, 387389(0):277 - 281, 2004. 13th International Conference on the Strength of Materials.

[9] K Srivastava, R Groeger, D Weygand, and P Gumbsch. Dislocation motion in tungsten: Atomistic input to discrete dislocation simulations. International Journal of Plasticity, 47:126 - 142, 2013.

[10] G Lu, N Kioussis, VV Bulatov, and E Kaxiras. Generalized-stacking-fault energy surface and dislocation properties of aluminum. Phys. Rev. B, 62:3099-3108, Aug 2000.

[11] E Clouet. The vacancyedge dislocation interaction in fcc metals: A comparison between atomic simulations and elasticity theory. Acta Materialia, 54(13):3543 - 3552, 2006. Selected Papers from the Meeting Micromechanics and Microstructure Evolution: Modeling, Simulation and Experiments held in Madrid/Spain, 1116 September 2005.

[12] L Nicola, AF Bower, KS Kim, Needleman A, and Van der Giessen E. Surface versus bulk nucleation of dislocations during contact. Journal of the Mechanics and Physics of Solids, 55(6):1120 - 1144, 2007.

[13] JA El-Awady. Unravelling the physics of size-dependent dislocation-mediated plasticity. Nat. Commun., 6:5926, 2015.

[14] M Huang, J Tong, and Z Li. A study of fatigue crack tip characteristics using discrete dislocation dynamics. International Journal of Plasticity, 54:229 - 246, 2014.

[15] LE Shilkrot, EM Ronald, and WA Curtin. Multiscale plasticity modeling: coupled atomistics and discrete dislocation mechanics. Journal of the Mechanics and Physics of Solids, 52(4):755 - 787, 2004.

[16] B Shiari, RE Miller, and WA Curtin. Coupled atomistic/discrete dislocation simulations of nanoindentation at finite temperature. Journal of Engineering Materials and Technology-Transactions of the ASME, 127(4):358-368, 2005.

[17] T Junge. Modelling Plasticity in Nanoscale Contact. PhD thesis, ENAC, Lausanne, 2014.

[18] J Cho, T Junge, JF Molinari, and G Anciaux. Toward a 3d coupled atomistic and discrete dislocation dynamics simulation: dislocation core structures and peierls stresses with several character angles in fcc aluminum. Advanced Modeling and Simulation in Engineering Sciences, 2(1):1-17, 2015.

[19] A Stukowski and K Albe. Extracting dislocations and non-dislocation crystal defects from atomistic simulation data. Modelling and Simulation in Materials Science and Engineering, 18(8):085001, 2010.

[20] FRN Nabarro. Dislocations in a simple cubic lattice. Proceedings of the Physical Society, 59(2):256, 1947.

[21] G Leibfried. Über den einfluß thermisch angeregter schallwellen auf die plastische deformation. Zeitschrift für Physik, 127(4):344-356, 1950.

[22] A Hikata, RA Johnson, and C Elbaum. Interaction of dislocations with electrons and with phonons. Phys. Rev. B, 4:674-674, Jul 1971

[23] JA Gorman, DS Wood, and T Vreeland. Mobility of dislocations in aluminum. Journal of Applied Physics, 40(2):833-841, 1969.

[24] JD Eshelby. Supersonic dislocations and dislocations in dispersive media. Proceedings of the Physical Society. Section B, 69(10):1013, 1956.

[25] W Atkinson and N Cabrera. Motion of a frenkelkontorowa dislocation in a one-dimensional crystal. Phys. Rev., 138:A763-A766, May 1965.

[26] S Ishioka. Steady Motion of a Dislocation in a Lattice. Journal of the Physical Society of Japan, 34:462, February 1973.

[27] S Ramos de Debiaggi and A Caro. Phonons radiated by moving dislocations in disordered alloys. J. Phys. France, 48(9):1499-1504, 1987.

[28] N Flytzanis, V Celli, and A Nobile. Motion of two screw dislocations in a lattice. Journal of Applied Physics, 45(12):5176-5181, 1974.

[29] J Frenkel and T Kontorowa. On the theory of plastic deformation and twinning. Physikalische Zeitschrift der Sowjetunion, 13:1, 1938.

[30] K Kang, VV. Bulatov, and W Cai. Singular orientations and faceted motion of dislocations in bodycentered cubic crystals. Proceedings of the National Academy of Sciences, 109(38):15174-15178, 2012.

[31] E Martínez, J Marian, A Arsenlis, M Victoria, and JM Perlado. Atomistically informed dislocation dynamics in FCC crystals. Journal of the Mechanics and Physics of Solids, 56(3):869 - 895, 2008.

[32] D Olmsted, GH Louis, WA Curtin, and RH Clifton. Atomistic simulation of dislocation mobility in $\mathrm{Al}, \mathrm{Ni}$ and $\mathrm{Al} / \mathrm{Mg}$ alloys. Modeling And Simulation in Materials Science and Engineering, 13(3):371-388, 2005 
[33] N Bhate, RJ Clifton, and R Phillips. Atomistic simulations of the motion of an edge dislocation in aluminum using the embedded atom method. AIP Conference Proceedings, 620(1):339-342, 2002.

[34] A Arsenlis, W Cai, M Tang, M Rhee, T Oppelstrup, G Hommes, T Pierce, and VV Bulatov. Enabling strain hardening simulations with dislocation dynamics. Modelling and Simulation in Materials Science and Engineering, 15(6):553, 2007.

[35] HM Zbib, TD Rubia, Moono Rhee, and JP Hirth. 3d dislocation dynamics: stressstrain behavior and hardening mechanisms in fcc and bcc metals. Journal of Nuclear Materials, 276(13):154 - 165, 2000.

[36] E Martínez and JP Hirth. Screw-dislocation constrictions in face-centered cubic crystals. Phys. Rev. B, 90:064102, Aug 2014.

[37] D Hull and D Bacon. Preface. In D Hull and DJ Bacon, editors, Introduction to Dislocations (Fifth Edition), pages ix - x. Butterworth-Heinemann, Oxford, fifth edition edition, 2011.

[38] MI Mendelev, MJ Kramer, CA Becker, and M Asta. Analysis of semi-empirical interatomic potentials appropriate for simulation of crystalline and liquid $\mathrm{Al}$ and $\mathrm{Cu}$. Philosophical Magazine, 88(12):1723-1750, 2008

[39] T Schneider and E Stoll. Molecular-dynamics study of a three-dimensional one-component model for distortive phase transitions. Phys. Rev. B, 17:1302-1322, Feb 1978.

[40] T Vreeland and KM Jassby. Temperature dependent viscous drag in close-packed metals. Materials Science and Engineering, 7(2):95 - 102, 1971.

[41] J Marian and A Caro. Moving dislocations in disordered alloys: Connecting continuum and discrete models with atomistic simulations. Phys. Rev. B, 74:024113, Jul 2006.

[42] Charles Kittel. Introduction to Solid State Physics. John Wiley \& Sons, Inc., New York, 6th edition, 1986.

[43] WK Liu, EG Karpov, and HS Park. Nano Mechanics and Materials. John Wiley and Sons, Ltd, 2006.

[44] EB Tadmor, RS Elliott, JP Sethna, RE Miller, and CA Becker. Knowledgebase of Interatomic Models (KIM). https://openkim.org, 2011.

[45] VV Bulatov, W Cai, J Fier, M Hiratani, G Hommes, T Pierce, M Tang, M Rhee, K Yates, and $\mathrm{T}$ Arsenlis. Scalable line dynamics in paradis. sc-conference.org/sc2004/schedule/pdfs/pap206.pdf.

[46] A Wolfenden. Multi-faulted dislocation loops in electron bombarded aluminium. Micron (1969), $4(3): 295-305,1972$. 